\title{
Surgical Treatment of a Rare Presentation of Bertolotti's Syndrome from Castellvi Type IV Lumbosacral Transitional Vertebra: Case Report and Review of the Literature
}

\author{
Ryan Adams $^{1}$ Sarah Herrera-Nicol ${ }^{1,2}$ Arthur L. Jenkins III ${ }^{1,2}$ \\ ${ }^{1}$ Department of Neurosurgery, Icahn School of Medicine at Mount \\ Sinai, The Mount Sinai Hospital, New York, New York, United States \\ 2 Department of Orthopedics, Icahn School of Medicine at Mount \\ Sinai, The Mount Sinai Hospital, New York, New York, United States \\ Address for correspondence Arthur L. Jenkins, MD, Department of \\ Neurosurgery, Icahn School of Medicine at Mount Sinai, The Mount \\ Sinai Hospital, 1468 Madison Avenue, Annenberg Building, Floor 8, \\ Room 90, New York, NY 10029, United States \\ (e-mail: arthur.jenkins@mountsinai.org).
}

J Neurol Surg Rep 2018;79:e70-e74.

\begin{abstract}
Keywords

- spine

- Bertolotti's syndrome

- lumbosacral transitional vertebrae

- minimally invasive surgery

- mechanical back pain

- radiating leg pain

- case report
\end{abstract}

Background Advancements in radiological imaging and diagnostic criteria enable doctors to more accurately identify lumbosacral transitional vertebrae (LSTV) and their association with back and L5 distribution leg pain. It is considered the most common congenital anomaly of the lumbosacral spine with an incidence between 4 and $35 \%$, ${ }^{3}$ although many practitioners describe 10 to $12 \%$ overall incidence. LSTVs include sacralization of the L5 vertebral body and lumbarization of the S1 segment while demonstrating varying morphology, ranging from broadened transverse processes to complete fusion. ${ }^{5}$ The most common types of LSTV that present with symptomatic Bertolotti's syndrome are the Castellvi type I and type II; type III and type IV variants rarely present with symptoms referable with confirmatory and provocative testing to the transitional vertebra itself, and therefore there is limited experience and no case reports of treatment toward this particular entity.

Case Description We illustrated a case of a 37 years old female in which a computed tomography scan demonstrated type III LSTV on the left and a type I anomaly on the right. The patient presented with right-sided leg pain and left-sided sacroiliac $(\mathrm{SI})$ region low back pain, worse with rotation and standing, for several years, and had been on daily narcotic pain medications for more than 2 years. The patient had temporary relief of her leg pain with a transverse/ALA injection on the right, but no improvement in her back pain, whereas a left-sided injection into the region around the type III interface on the left did transiently alleviate her SI pain without improvement in her leg pain. We proposed that this particular anomaly induced mechanical back pain on the left side by flexion of the bone bridge (a form of stress-fracture, with associated sclerotic changes in the interface in the transverse/ALA junction) with associated irritation of the right L5 nerve from the type I anomaly on the right in conjunction with her typical radiating leg pain on the right. A patent, but somewhat hypoplastic L5/S1 disk space was also present. Nonsegmental pedicle screw instrumentation with low-profile screws was implanted on the right side with fusion induced using allograft and off label use of infuse rh-BMP2 bone graft received

January 3, 2018 accepted after revision May 14, 2018
DOI https://doi.org/

10.1055/s-0038-1667172. ISSN 2193-6358. (c) 2018 Georg Thieme Verlag KG Stuttgart · New York
License terms

c) $(1) \$$ 
substitute, and the patient was discharged the same day. The patient noted immediate improvement in her preoperative symptoms, and by 2 weeks after her surgery noted complete resolution of the preoperative symptoms, and required no narcotic medications to control her incisional pain.

Conclusion Patients who present with symptoms consistent with Bertolotti's syndrome, even if they have a type III or type IV LSTV, should be considered for surgical treatment of their LSTV. These patients can respond well, even if symptoms have been present for years. Given the prevalence of these anatomic variants in the general population (10-12\% in most series), Bertolotti's syndrome should be considered in the differential diagnosis of any patient with a presentation of L5 radiculopathy and/or back pain.

\section{Introduction}

Lumbosacral transitional vertebrae (LSTV) are increasingly recognized as a common anatomical variant associated with altered patterns of degenerative spine changes. ${ }^{1,2}$ Due to possible other comorbidities present in the spine, the diagnosis of Bertolotti's syndrome is not straightforward thus making it a diagnosis of exclusion. ${ }^{3}$ Patients with symptomatic LSTV, also known as Bertolotti's syndrome, typically present with some combination of unilateral or bilateral symptoms. The manifestation of these symptoms is presumably related to the bone-on-bone contact at the lumbosacral or the lumboiliac interfaces. This presents itself usually as some combination of SI region pain, hip pain, groin pain, or L5 radicular pain stemming from irritation and inflammation around the portion of the L5 nerve that runs past the interface after it leaves the foramen. ${ }^{4}$ Retrospective analysis of patients presented with symptomatic LSTV in the practice of Dr. Arthur Jenkins implicated five main pain loci. These loci can be presented symmetrically or asymmetrically in regions such as the groin, hip, SI joint, leg, and back. Pain loci in the back can also stem from the midline relative to the lumbosacral level. While these pain loci can manifest from LSTV, further clinical assessments are necessary to assume a positive or negative correlation to other spinal comorbidities. Although the exact cause of this syndrome is unclear, the following etiologies have been put forward: (1) articulation of the transverse process and sacral ALA, (2) degeneration of the intervertebral disk at adjacent levels, (3) facet joint contralateral to a unilateral fused or articulating LSTV, and (4) extraforaminal stenosis secondary to the presence of a hypertrophied transverse process. ${ }^{5}$ Castellvi et al classified LSTV into four types (-Fig. 1). ${ }^{6}$ Type I includes unilateral (Ia) or bilateral (Ib) dysplastic transverse processes, measuring at least $19 \mathrm{~mm}$ in width (craniocaudal dimension). Type II exhibits incomplete unilateral (IIa) or bilateral (IIb) lumbarization/sacralization with an enlarged transverse process that has a diarthrodial joint between itself and the sacrum.
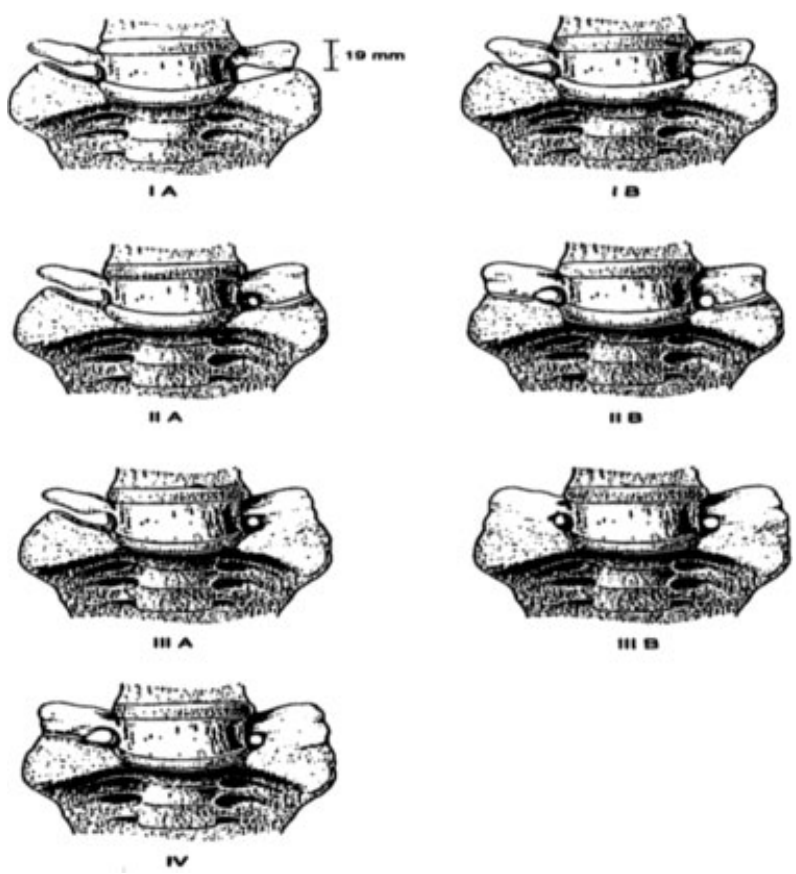

Fig. 1 Schematic presentation of the classification of unilateral and bilateral lumbosacral transitional vertebrae according to Castellvi. ${ }^{6}$ 
Type III LSTV describes unilateral (IIIa) or bilateral (IIIb) lumbarization/sacralization with complete osseous fusion of the transverse process(es) to the sacrum. Type IV involves a unilateral type II transition with a type III on the contralateral side. 6,7

We present a patient with a type IV LSTV who was symptomatic on both sides with differing symptoms between the right and left sides. The long-standing incidence of low back pain resulted in difficulties to weight-bear on the right side and to sit down. The patient underwent extensive conservative management including acupuncture and 8 months of physical therapy prior to electing surgery for a L5/S1 posterior lumbar interbody fusion. A recent right-sided L5 transverse process/sacral ALA injection induced radiation of her leg pain in an S1 distribution. Subsequent left-sided L5 transverse process/sacral ALA injections helped her to weightbear more on her right leg. In addition to the ALA injections, she underwent a right peroneal nerve tendon repair in 2014 which did not alleviate the pain in the region or her ability to weight-bear. With the correct diagnosis, we performed a minimally invasive fusion of her unfused side, with near complete resolution of her preoperative symptoms.

\section{Case Description}

The patient elected to undergo minimally invasive surgery for mechanical back pain in conjunction with radiating leg pain. A presurgical computed tomography scan demonstrated type III
LSTV on the left and a type I anomaly on the right side ( - Figs. 2 and 3 ). The patient noted an alleviation of her symptoms through bilateral Bertolotti's injections, but failed to improve with both transforaminal epidural steroid injections at L4 to 5 and L5/S1 and SI joint injections.

Upon presurgical post-anesthesia care unit arrival, standard-of-care treatment was implemented. The patient was anesthetized, intubated, and then placed into a posterior prone position in the operating room (OR) suite. The marked incision was infiltrated with $0.5 \%$ Marcaine, $1: 200,000$ epinephrine, and incised with a \#10 blade scalpel superficially. A Bovie cautery was then utilized to infiltrate through the dermis and then subsequently down through the lumbar dorsal fascia. The Bovie cautery was then dissected down through the paraspinal muscles onto the left L5 transverse process. Once the L5 transverse process was palpated, the METRx first dilator was passed through the incision and docked on the midpoint of the L5 transverse process. This process was confirmed under lateral fluoroscopy. The subsequently larger dilators were then passed until a METRxtube working channel was able to be passed over the last dilator and attached to the table-mounted array. This was then secured after lateral fluoroscopy confirmed that the trajectory of the working channel was now aiming toward the bottom half of the transverse process overlapping the left transverse ALA junction. After securing the working channel and removing the dilators, the operating microscope was then brought into the field. Standard microsurgical
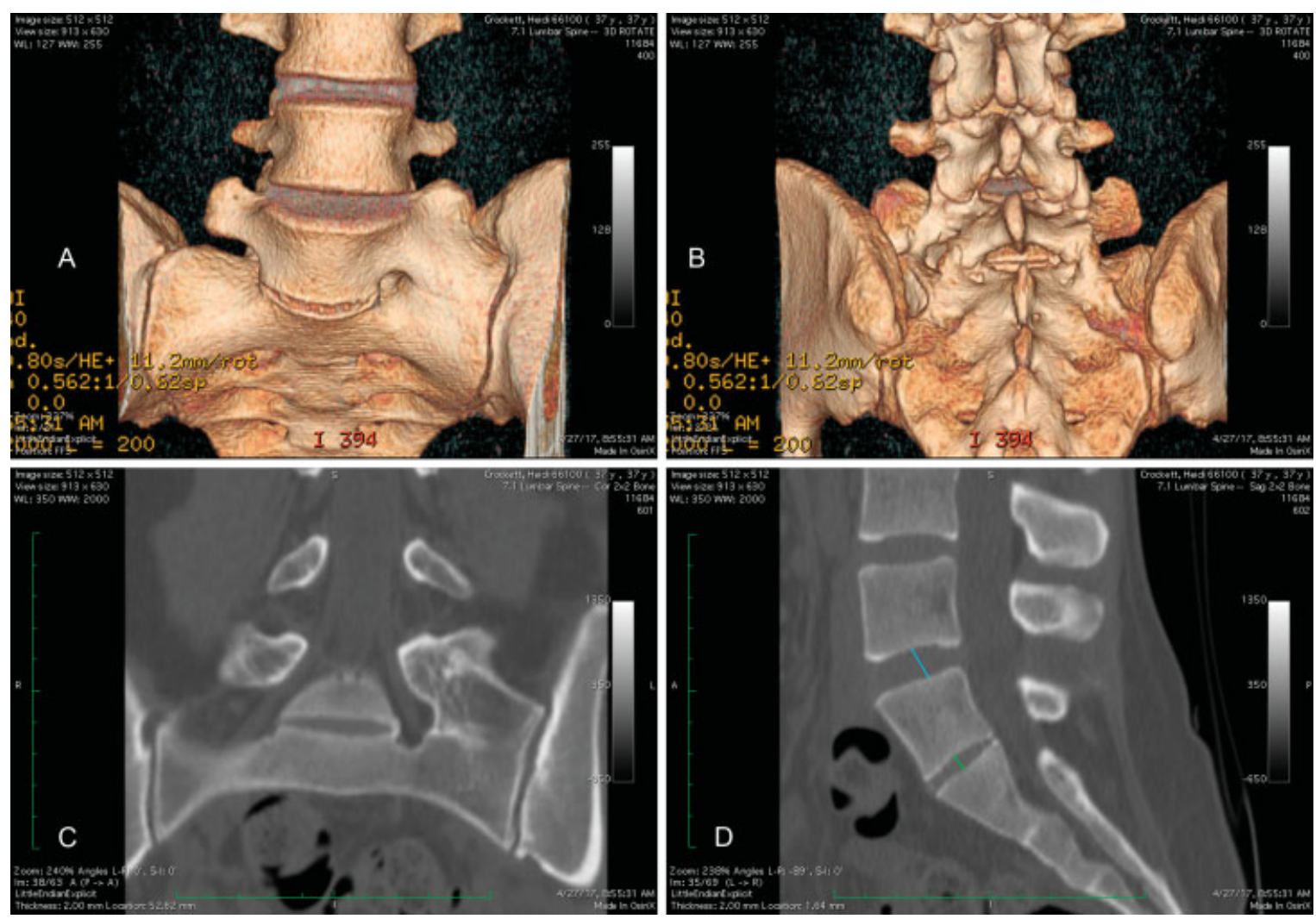

Fig. 2 (A) Anterior view of three-dimensional reconstruction of the patient's pseudoarticulation present between L5 and S1. (B) Posterior view. (C) Anterior computed tomography scans depicting scoliotic changes induced by the type III pseudoarticulation. (D) Sagittal view detailing vertebral disk height of the L4/L5 interface $(10.0 \mathrm{~mm})$ and L5/S1 interface $(5.3 \mathrm{~mm})$. 

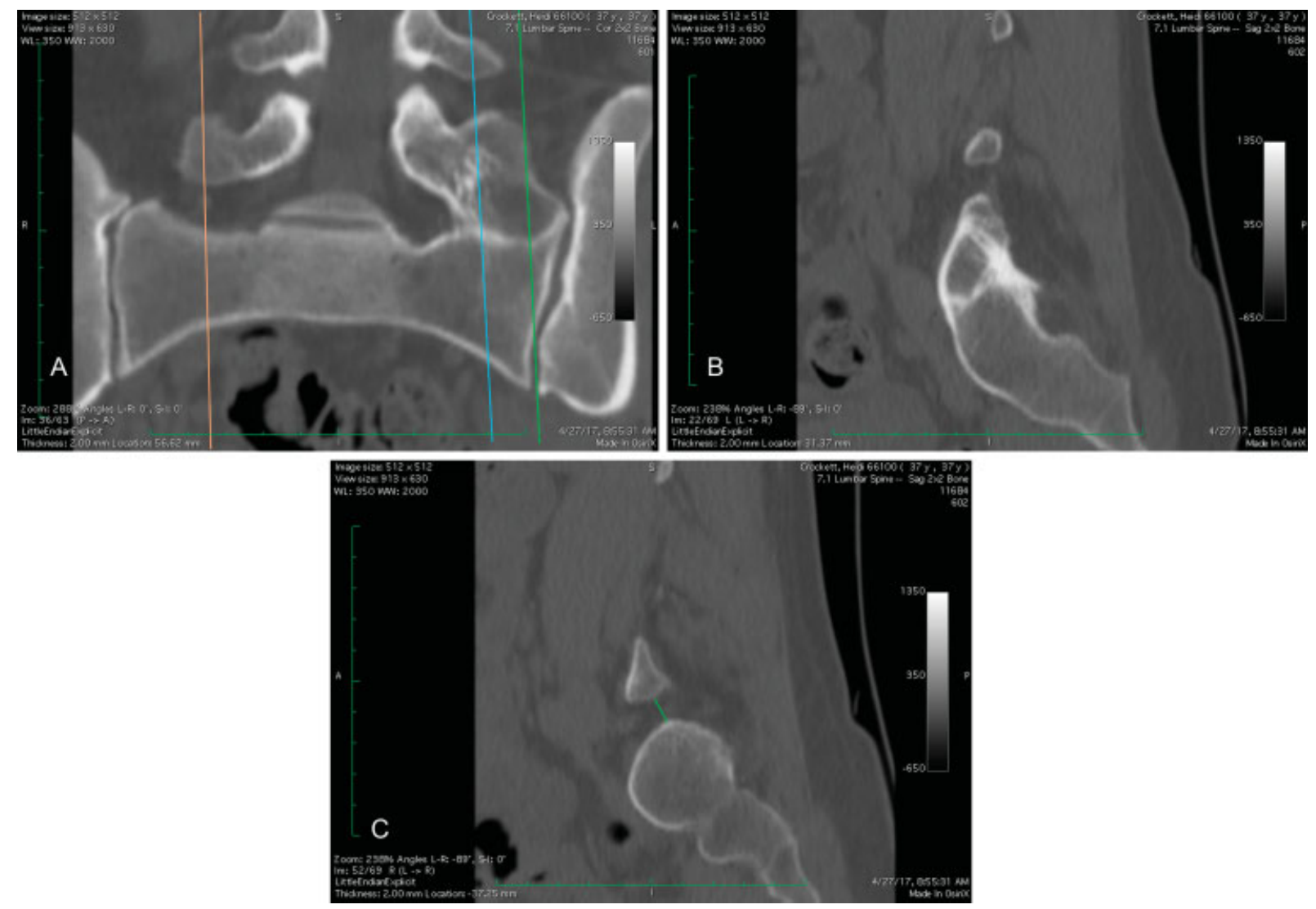

Fig. 3 (A) Anterior computed tomography scan depicting sagittal imaging locations. (B) Sagittal view of the type I formation depicting $7.8 \mathrm{~mm}$ of space between L5 and S1 (C) type III pseudoarticulation showing the spontaneous fusion between L5 and S1.

dissection techniques were utilized to aim the working channel medially toward the right transverse pedicle junction which was then identified and palpated. Subsequently, the L5/S1 neural foramen was identified as was the medial aspect of the L5/S1 facet joint on the right. The muscular attachments to the facet, the $\mathrm{L} 5$ transverse process, and the ALA were removed with the Bovie cautery. The facet capsule of $\mathrm{L} 5 / \mathrm{S} 1$ on the right was removed and the joint partially decorticated with the TAC- 125 bit. The entry point on the L5 transverse/pedicle junction was then identified with lateral fluoroscopy, and an entry point created. The METRx-tube was redirected in a parallel fashion to the pedicle trajectory and the gearshift pedicle probe was passed, under fluoroscopic guidance, into the vertebral body of L5 through the pedicle of L5. A ball-tipped pedicle probe was utilized to confirm no violations of the cortex medially, laterally, rostral, caudally, or deep were created. A small amount of Surgifoam was passed into the tract, and the $5.5 \mathrm{~mm}$ tap was then implanted down the tract under fluoroscopic guidance. The entry point on the S1 ALA/facet junction was identified with lateral fluoroscopy, and a TAC-125 bit was used to infiltrate the location. The tube was subsequently redirected to a parallel position relative to the pedicle trajectory and the gearshift pedicle probe passed, under fluoroscopic guidance, into the vertebral body of S1 through the pedicle of S1. A balltipped pedicle probe then confirmed no violations of the cortex medially, laterally, rostral, caudally, or deep. Surgi- foam was injected into the tract, and a $5.5 \mathrm{~mm}$ tap was implanted, under fluoroscopic guidance. The wound was irrigated with antibiotic saline prior to drilling the cortex off the inferior half of the $\mathrm{L} 5$ transverse process and the sacral ALA utilizing a TAC-125 bit. Hemostasis was obtained with Surgifoam and bipolar cautery. The bone chips from drilling were left in place. Half of the crushed cancellous chips were then passed down the tube over the intertransverse region spanning from L5 to ALA. Screws $(6.5 \times 35 \mathrm{~mm})$ were then implanted down the tube, each time redirecting the tube in a parallel fashion to the original trajectories for each pedicle. After achieving excellent purchase with both screws, a $30 \mathrm{~mm}$ rod was positioned down the tube into each screw's head, and locking caps applied. The field was covered with a circumferential drape, and an ISO-C 3D reconstruction performed, which showed both screws in excellent position. The locking caps were subsequently tightened to their final torque tension and the remaining extensions cracked off and collected. An Infuse Extra-Small kit sponge was divided in two, with half being placed over the L5/S1 facet decortication, and the other half positioned over the first component of graft lateral to the hardware. The graft material was then packed over the Infuse sponge with the remainder of the graft positioned down the tube under the muscle within the intertransverse region. The working channel was then gradually removed and hemostasis obtained in the paraspinal muscles with electrocautery. Once the working channel was 
removed completely, a UR-6 0 Vicryl suture was utilized to close the lumbar dorsal fascia in a watertight fashion using figure-of-eight sutures. The wound was then irrigated again with antibiotic-containing saline prior to juxtaposing the Scarpa's fascia using 0 Vicryl suture. The subcuticular layer was closed using interrupted inverted 3-0 Vicryl suture. Dermabond was then applied in two layers. Once the second layer of Dermabond had dried, the drapes were removed and the patient was relocated from the OR table to the OR stretcher and extubated. The patient was found to be moving all four extremities well, with no new complaints. Postsurgical analysis demonstrated that the patient tolerated the procedure well without any apparent complications.

\section{Discussion}

Although not all patients with LSTV will develop Bertolotti's syndrome, patients with all types of LSTV can present with symptoms and a careful evaluation of the LSTV as a possible source of pain needs to be undertaken in this patient population.

In this case, a combination of lack of response to transforaminal epidural steroid injection, facet, and SI joint injection coupled with successful temporary analgesia of different components of her pain assisted with increasing our confidence that both symptoms were coming from the anomaly. She had alleviation of the left-sided SI pain from injection of the fused left-sided bone bridge, and alleviation of the rightsided L5 symptoms from the injection of the right-sided TP/ ALA interface. We surmised that her left-sided bone bridge was undergoing a stress response, akin to a stress fracture, due to the presence of motion at the hypoplastic disk, and the anatomically functional facet joint and TP-ALA gap on the right, but the flexion was enough to allow for irritation on the right $\mathrm{L} 5$ nerve. Given the responses of the injections and the surgical treatment, this hypothesis seems to hold water.

\section{Conclusion}

Patients, who present with symptoms consistent with Bertolotti's syndrome even if they have a type III or type IV LSTV, should be considered for surgical treatment of their LSTV. These patients can respond well, even if symptoms have been present for years. Given the prevalence of these anatomic variants in the general population (10-12\% in most series),
Bertolotti's syndrome should be considered in the differential diagnosis of any patient with a presentation of L5 radiculopathy and/or back pain.

\section{Consent}

Informed consent was obtained from the patient presented in this case report.

Conflicts of Interest

None to disclose.

\section{Statement of Nonduplication}

I, Arthur L. Jenkins III, certify that this manuscript is a unique submission and is not being considered for publication, in part or in full, with any other source in any medium.

\section{Funding Source}

This research did not receive any specific grant from funding agencies in the public, commercial, or not-forprofit sectors.

\section{References}

1 Josiah DT, Boo S, Tarabishy A, Bhatia S. Anatomical differences in patients with lumbosacral transitional vertebrae and implications for minimally invasive spine surgery. J Neurosurg Spine 2017;26(02):137-143

2 Konin GP, Walz DM. Lumbosacral transitional vertebrae: classification, imaging findings, and clinical relevance. AJNR Am J Neuroradiol 2010;31(10):1778-1786

3 Paudel B, Kim HS, Jang JS, et al. Percutaneous full endoscopic treatment of Bertolotti syndrome: a report of three cases with technical note. J Neurol Surg A Cent Eur Neurosurg 2017;78(06): 566-571

4 Porter NA, Lalam RK, Tins BJ, Tyrrell PN, Singh J, Cassar-Pullicino VN. Prevalence of extraforaminal nerve root compression below lumbosacral transitional vertebrae. Skeletal Radiol 2014;43(01): 55-60

5 Takata Y, Sakai T, Higashino K, et al. Minimally invasive microendoscopic resection of the transverse process for treatment of low back pain with Bertolotti's syndrome. Case Rep Orthop 2014; 2014(14):613971

6 Jancuska JM, Spivak JM, Bendo JA. A review of symptomatic lumbosacral transitional vertebrae: Bertolotti's syndrome. Int J Spine Surg 2015;9:42

7 Pandit S,Monalisa, Sapkota S, Kumar S. A case report of a variant of lumbo-sacral transition vertebrae: Castellvi type Iia sacralisation. Int J Anat Res 2015;3(03):1285-1288 